\title{
Avaliação da percepção dos alunos da disciplina de endodontia sobre o uso do Ambiente Virtual de Aprendizagem (Moodle). Uso do questionário de auto-avaliação COLLES
}

\author{
Ivana Maria Zaccara Cunha-Araújo*, Juan Ramon Salazar-Silva**, Fábio Luiz Cunha \\ D’Assunção**, Ângelo Brito Pereira de Melo** \\ * Cirurgiã-dentista, mestranda em Saúde Coletiva - Odontologia, \\ Universidade Federal do Rio Grande do Norte \\ ** Professor Adjunto de Endodontia da Universidade Federal | \\ da Paraíba
}

\section{RESUMO}

A Disciplina de Endodontia I da Universidade Federal da Paraíba (UFPB) implantou a partir da turma 2008.1, o sistema de gerenciamento de cursos, mediante Plataforma Moodle. Ao final do período é empregado um questionário de avaliação disponível no ambiente Moodle. O objetivo deste trabalho foi analisar os resultados do questionário COLLES do tipo "experiência efetiva", aplicados aos 57 alunos das turmas 2008.2 e 2009.1 da disciplina de Endodontia I. O questionário COLLES permite avaliar os quesitos: Relevância, Reflexão Crítica, Interação, Apoio dos Tutores, Apoio dos Colegas e Compreensão. A partir das respostas obtidas foram gerados gráficos com resultados numéricos que posteriormente foram analisados quantitativamente. Os resultados revelaram excelente relevância em que 73,6\% dos alunos relatam a importância do aprendizado para prática da profissão e 68,4\% demonstraram a melhora no seu desempenho. Já em relação à reflexão crítica, $41 \%$ refletem criticamente com frequência sobre os conteúdos do curso e 43,8\% refletem sobre como está sendo o seu aprendizado. A interatividade entre os alunos apresenta-se deficiente, pois uma pequena parcela $(21 \%)$ se dispõe a explicar suas ideias aos outros participantes. No apoio dos tutores, apresentou frequente estímulo a reflexão e encorajamento à participação; já no apoio entre os colegas, houve baixa frequência de elogio e estima as contribuições. Os alunos também apresentaram frequente compreensão das mensagens dos participantes $(50,8 \%)$ e dos tutores $(57,8 \%)$. Conclui-se que existe necessidade de estimular o apoio entre os alunos e interação de suas ideias para que haja um melhor aproveitamento dentro do meio de ensino à distância.

\section{DESCRITORES}

Educação à Distância. Materiais de Ensino. Ensino. Educação em Odontologia. Endodontia.

O processo ensino-aprendizagem constitui o fundamento da prática docente universitária a qual tem como eixo principal o aluno. No entanto, paralelamente ou como parte inseparável desse processo, a avaliação é a ferramenta que fornece informações oportunas que buscam investigar se a aprendizagem está sendo conseguida ou não, bem como nos indica a retomada do caminho que busca o objetivo final. ${ }^{1}$

Avaliação é um processo contínuo de pesquisas que visa interpretar os conhecimentos, habilidades e atitudes dos alunos, tendo em vista mudanças esperadas no comportamento, propostas nos objetivos educacionais, a fim de que haja condições de decidir sobre alternativas do planejamento do trabalho do professor e da escola como um todo. ${ }^{14}$ A tarefa de avaliar, realizada de forma contínua busca a retroalimentação do processo de aprendizagem, na tentativa de concretizar o aprendizado do aluno. Para Masetto $^{10}$ (1992) este processo constitui uma das atividades pedagógicas mais difíceis de realizar.

O processo de avaliação deverá estar voltado para o desempenho do aluno, verificando se realiza 
Avaliação da percepção dos alunos da disciplina de endodontia sobre o uso do Ambiente Virtual de Aprendizagem (Moodle). Uso do questionário de auto-avaliação COLLES • Cunha-Araújo IMZ, Salazar-Silva JR, D’Assunção FLC, Melo ABP

adequada ou inadequadamente o que foi planejado. Em outras palavras este processo busca o acompanhamento do aluno no seu processo de desenvolvimento. ${ }^{1}$

A avaliação do processo ensino-aprendizagem é um assunto polêmico em todas as áreas da educação. Nas disciplinas das ciências da saúde observa-se, uma carência de instrumentos de avaliação por parte do professor, tornando esse processo subjetivo e sujeito as reclamações por parte dos discentes, ainda o devido acompanhamento diário do discente fica prejudicado. ${ }^{5}$

Por outro lado, o Ensino a Distância (EaD) vem sendo aplicado gradativamente nos cursos de conteúdos que permitam o desenvolvimento do aspecto cognitivo sem a presença física de um tutor. Especificamente, na área da saúde, alguns cursos vêm utilizando-se do suporte a distância. ${ }^{16}$

Ambientes de aprendizagem online são uma proposta de ensino à distância que se apresentam cada vez mais viáveis, graças à inclusão tecnológica de grande número das instituições educacionais das grandes cidades. Segundo dados do Censo da Educação Superior de 2010, no período de 2006 a 2010 houve um aumento em número de cursos e no número de matrículas, de forma considerável para os cursos na modalidade EaD. Em 2005, os alunos de EaD representavam 2,6\% do universo dos estudantes. Em 2010 essa participação passou a ser de $14,1 \% .^{9}$

Esses percentuais propõem desafios tanto para docentes quanto para discentes e, dentre eles, pode-se citar a necessidade de:

- superar a passividade dos estudantes, reflexo de anos de uma pedagogia transmissiva;

- aprender a lidar com uma nova demanda comunicacional, provocada pela evolução da tecnologia e pela convergência das mídias;

- promover o desenvolvimento de comunidades;

- quebrar vários paradigmas;

- procurar novas formas de avaliação. ${ }^{15}$

A utilização da tecnologia da informação, para aplicação dos princípios pedagógicos, tem sido centrada na criação de ferramentas computacionais em que os estudantes possam manipular para completar a sua memória e inteligência na construção de modelos mentais mais exatos. ${ }^{2,7}$

Dentro desse contexto, a Disciplina de Endodontia I da UFPB, implantou a partir da turma 2008.1, o Ambiente Virtual de Aprendizagem (AVA) também conhecido como sistema de gerenciamento de cursos (SGC), mediante o uso da Plataforma Moodle que procura buscar um melhor resultado do processo ensino e do aprendizado usando as vantagens da internet sem dispensar a necessidade do professor. Dessa forma, a disciplina de Endodontia I tornou-se uma disciplina presencial com apoio à distância. ${ }^{6}$

Essa ferramenta, objetiva integrar as atividades presenciais com atividades vinculadas ao Ensino à Distância, permitindo, que o material utilizado em sala de aula esteja disponível ao aluno para que este o acompanhe, podendo também consultar páginas web indicadas pelos professores, verificar planos de aula, consultar manuais práticos de procedimentos para acompanhamento em trabalhos laboratoriais, participar na elaboração do glossário de termos endodônticos, participar de fórum de discussão, de chats, plantão tira-dúvidas e de resolução de questões. Para melhor monitoramento do ensino, são disponibilizados aos tutores:

- número de acessos por aluno,

- páginas mais visitadas e

- tempo de permanência nas mesmas. ${ }^{11,16}$

O Moodle cumpre com os objetivos de sua criação: viabilizar o acesso à aprendizagem à distância por comunidades de usuários que privilegiam o discurso colaborativo em suas interações. O sistema contém alguns tipos de questionários de avaliação de cursos, específicos para ambientes de aprendizagem virtuais, conhecidos como ATTLS (Attitudes to Thinking and Learning Survey), COLLES (Constructivist On-Line Learning Environment Survey) e Incidentes críticos.

O COLLES se propõe a avaliar as percepções dos alunos a respeito:

- da relevância do curso de que estão participando (se está atendendo às suas expectativas);

- a qualidade da interação no ambiente;

- a qualidade das discussões no que se refere ao pensamento crítico e reflexivo;

- a qualidade do suporte oferecido pelo tutor e

- se significados são construídos por meio de saber conectado entre os alunos e entre os alunos e tutores. ${ }^{17}$

Diante do exposto, o objetivo deste trabalho foi analisar os resultados do questionário COLLES do tipo "experiência efetiva", aplicados aos 57 alunos das turmas 2008.2 e 2009.1 da disciplina de Endodontia I do curso de Odontologia da Universidade 
Avaliação da percepção dos alunos da disciplina de endodontia sobre o uso do Ambiente Virtual de Aprendizagem (Moodle). Uso do questionário de auto-avaliação COLLES • Cunha-Araújo IMZ, Salazar-Silva JR, D’Assunção FLC, Melo ABP

Federal da Paraíba, que obtiveram ensino presencial suportado pela ferramenta Moodle.

\section{MATERIAL E MÉTODOS}

No primeiro dia de aula presencial de cada período letivo são apresentados os objetivos da disciplina, seu conteúdo programático, bem como os alunos são instruídos sobre o uso e as facilidades da Plataforma Moddle para o aprendizado (Figura 1).

Ao final do período é aplicado um questionário de avaliação disponível no ambiente Moodle, que permite a reflexão sobre os processos de aprendizagem.

O questionário de auto-avaliação COLLES é formado por 24 declarações distribuídas em 6 grupos:

- Relevância,

- Reflexão crítica,

- Interação,

- Apoio dos Tutores,

- Apoio dos Colegas e

- Compreensão.

Cada um deles relativo a um ponto crucial de avaliação da qualidade do processo de aprendizagem no ambiente virtual. O sub-tipo de questionário "experiência efetiva" que foi aplicado no final do curso, é uma boa ferramenta de avaliação do professor e dos monitores, pois mostra a relação entre as expectativas dos participantes e sua experiência efetiva.

A partir das respostas obtidas foram geradas tabe- las com resultados numéricos de cada uma das 24 declarações dos 57 alunos que posteriormente foram analisados quantitativamente.

\section{RESULTADOS}

Os resultados desta avaliação estão dispostos nas tabelas 1 a 6.

\section{DISCUSSÃo}

O uso do ambiente virtual de aprendizagem, nos cursos de odontologia, traz a oportunidade do uso de ferramentas tecnológicas, de modo a se estabelecer uma adequada interação entre o professor e o aluno participando ativamente das atividades do grupo, em discussões de tópicos relevantes, discussões que têm como objetivo comum a transformação de suas ações através de reflexões sobre a prática pedagógica. ${ }^{2}$ Além do mais, a modalidade semi-presencial, está sedimentada nas Diretrizes Curriculares Nacionais dos Cursos de Graduação em Odontologia, que estabelece na competência Comunicação, que os profissionais de saúde devem ser acessíveis as tecnologias de comunicação e informação. ${ }^{3}$ Já, a Portaria no. 4.059 de 10 de dezembro de 2004 regulamenta as disciplinas denominadas semi-presenciais e sugere que essas não ultrapassem $20 \%$ da carga total do curso. ${ }^{4}$

O emprego da tecnologia da informação aplicado ao ensino odontológico continua sendo um grande desafio mundial. O aumento da distribuição dos softwares livres para gerenciamento de cursos junta-
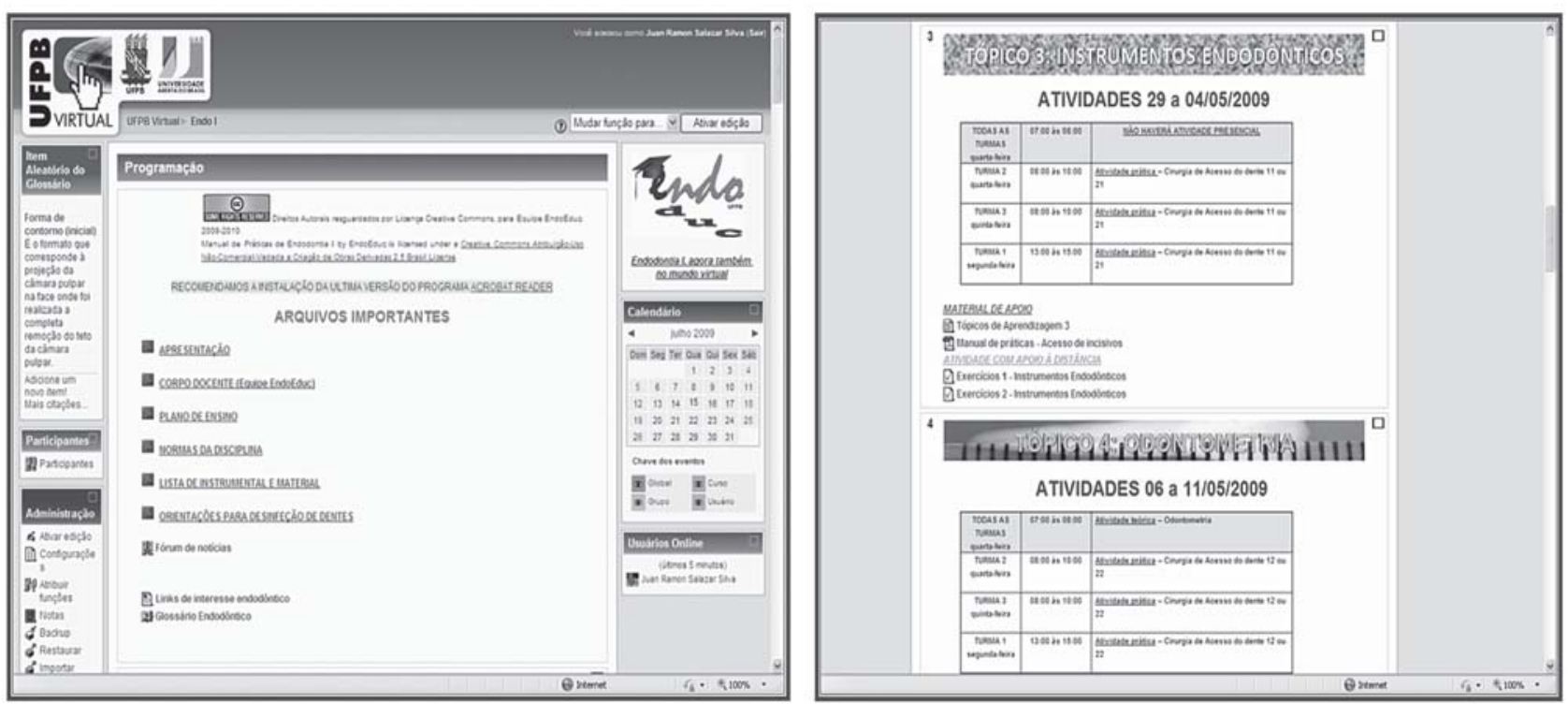

Figura 1 - Páginas da Disciplina de Endodontia na Plataforma Moodle. Página principal (esquerda); página das atividades programadas (direita). 
Avaliação da percepção dos alunos da disciplina de endodontia sobre o uso do Ambiente Virtual de Aprendizagem (Moodle). Uso do questionário de auto-avaliação COLLES • Cunha-Araújo IMZ, Salazar-Silva JR, D’Assunção FLC, Melo ABP

Tabela 1 - Valores numéricos referentes a relevância do processo de aprendizagem no ambiente virtual.

\begin{tabular}{|l|c|c|c|c|c|}
\hline \multicolumn{1}{|c|}{ Perguntas } & Quase nunca & Raramente & $\begin{array}{c}\text { Algumas } \\
\text { vezes }\end{array}$ & Frequentemente & $\begin{array}{c}\text { Quase } \\
\text { sempre }\end{array}$ \\
\hline $\begin{array}{l}\text { A aprendizagem é focalizada em } \\
\text { assuntos que me interessam? }\end{array}$ & 0 & 2 & 14 & 24 \\
\hline $\begin{array}{l}\text { O que eu estou aprendendo é importante } \\
\text { para prática da minha profissão? }\end{array}$ & 0 & 1 & 0 & 14 \\
\hline $\begin{array}{l}\text { Eu aprendo como fazer para melhorar o } \\
\text { meu desempenho profissional? }\end{array}$ & 0 & 0 & 6 & 12 \\
\hline $\begin{array}{l}\text { O que eu aprendo tem boas conexões com } \\
\text { a minha atividade profissional? }\end{array}$ & 0 & 1 & 1 & 19 \\
\hline
\end{tabular}

Tabela 2 - Valores numéricos referentes a reflexão crítica dos alunos durante o processo de aprendizagem no ambiente virtual.

\begin{tabular}{|l|c|c|c|c|c|}
\hline \multicolumn{1}{|c|}{ Perguntas } & Quase nunca & Raramente & $\begin{array}{c}\text { Algumas } \\
\text { vezes }\end{array}$ & Frequentemente & $\begin{array}{c}\text { Quase } \\
\text { sempre }\end{array}$ \\
\hline \multicolumn{1}{|c|}{ Eu reflito sobre como eu aprendo? } & 1 & 2 & 14 & 25 & 15 \\
\hline $\begin{array}{l}\text { Faço reflexões críticas sobre minhas } \\
\text { próprias ideias? }\end{array}$ & 0 & 5 & 20 & 23 & 9 \\
\hline $\begin{array}{l}\text { Faço reflexões críticas sobre as ideias dos } \\
\text { outros participantes? }\end{array}$ & 3 & 7 & 23 & 16 \\
\hline $\begin{array}{l}\text { Faço reflexões críticas sobres os conteúdos } \\
\text { do curso? }\end{array}$ & 1 & 1 & 17 & 24 & 8 \\
\hline
\end{tabular}

Tabela 3 - Valores numéricos referentes a interatividade durante o processo de aprendizagem no ambiente virtual.

\begin{tabular}{|l|c|c|c|c|c|}
\hline \multicolumn{1}{|c|}{ Perguntas } & Quase nunca & Raramente & $\begin{array}{c}\text { Algumas } \\
\text { vezes }\end{array}$ & Frequentemente & $\begin{array}{c}\text { Quase } \\
\text { sempre }\end{array}$ \\
\hline $\begin{array}{l}\text { Eu explico minhas ideias para os outros } \\
\text { participantes? }\end{array}$ & 4 & 10 & 23 & 12 & 8 \\
$\begin{array}{l}\text { Peço aos outros alunos explicações sobre } \\
\text { as ideias deles? }\end{array}$ & 3 & 3 & 18 & 23 & 10 \\
$\begin{array}{l}\text { Os outros participantes me pedem explica- } \\
\text { ções sobre as minha ideias? }\end{array}$ & 4 & 4 & 27 & 19 \\
$\begin{array}{l}\text { Os outros participantes reagem as minhas } \\
\text { ideias? }\end{array}$ & 3 & 14 & 23 & 14 \\
\hline
\end{tabular}

Tabela 4 - Valores numéricos referentes ao apoio dos tutores no processo de aprendizagem no ambiente virtual.

\begin{tabular}{|c|c|c|c|c|c|}
\hline \multicolumn{6}{|c|}{ Apoio dos Tutores } \\
\hline Perguntas & Quase nunca & Raramente & $\begin{array}{l}\text { Algumas } \\
\text { vezes }\end{array}$ & Frequentemente & $\begin{array}{l}\text { Quase } \\
\text { sempre }\end{array}$ \\
\hline O tutor me estimula a refletir? & 2 & 0 & 11 & 27 & 17 \\
\hline O tutor me encoraja a participar? & 1 & 1 & 13 & 23 & 19 \\
\hline $\begin{array}{l}\text { O tutor ajuda a melhorar a qualidade dos } \\
\text { discursos? }\end{array}$ & 2 & 5 & 18 & 16 & 16 \\
\hline $\begin{array}{l}\text { O tutor ajuda a melhorar o processo de } \\
\text { reflexão crítica? }\end{array}$ & 4 & 5 & 8 & 26 & 14 \\
\hline
\end{tabular}


Avaliação da percepção dos alunos da disciplina de endodontia sobre o uso do Ambiente Virtual de Aprendizagem (Moodle). Uso do questionário de auto-avaliação COLLES • Cunha-Araújo IMZ, Salazar-Silva JR, D’Assunção FLC, Melo ABP

Tabela 5 - Valores numéricos referentes ao apoio dos colegas no processo de aprendizagem no ambiente virtual.

\begin{tabular}{|c|c|c|c|c|c|}
\hline \multicolumn{6}{|c|}{ Apoio dos Colegas } \\
\hline Perguntas & Quase nunca & Raramente & $\begin{array}{l}\text { Algumas } \\
\text { vezes }\end{array}$ & Frequentemente & $\begin{array}{l}\text { Quase } \\
\text { sempre }\end{array}$ \\
\hline $\begin{array}{l}\text { Os outros participantes me encorajam a } \\
\text { participar? }\end{array}$ & 7 & 7 & 14 & 21 & 8 \\
\hline $\begin{array}{l}\text { Os outros participantes elogiam as minhas } \\
\text { contribuições? }\end{array}$ & 8 & 7 & 18 & 15 & 9 \\
\hline $\begin{array}{l}\text { Os outros participantes estimam as minhas } \\
\text { contribuições? }\end{array}$ & 8 & 6 & 21 & 11 & 11 \\
\hline $\begin{array}{l}\text { Os outros participantes demonstram } \\
\text { empatia quando me esforco para aprender? }\end{array}$ & 6 & 11 & 11 & 17 & 12 \\
\hline
\end{tabular}

Tabela 6 - Valores numéricos referentes a compreensão das mensagens no ambiente virtual.

\begin{tabular}{|l|c|c|c|c|c|}
\hline \multicolumn{1}{|c|}{ Perguntas } & Quase nunca & Raramente & $\begin{array}{c}\text { Algumas } \\
\text { vezes }\end{array}$ & Frequentemente & $\begin{array}{c}\text { Quase } \\
\text { sempre }\end{array}$ \\
\hline $\begin{array}{l}\text { Eu compreendo bem as mensagens dos } \\
\text { outros participantes? }\end{array}$ & 2 & 1 & 11 & 29 \\
\hline $\begin{array}{l}\text { Os outros participantes compreendem bem } \\
\text { as minhas mensagens? }\end{array}$ & 2 & 1 & 13 & 31 & 14 \\
\hline $\begin{array}{l}\text { Eu compreendo bem as mensagens do } \\
\text { tutor? }\end{array}$ & 2 & 0 & 5 & 33 \\
$\begin{array}{l}\text { O tutor compreende bem as minhas } \\
\text { mensagens? }\end{array}$ & 3 & 2 & 12 & 26 & 17 \\
\hline
\end{tabular}

mente com o desenvolvimento de ferramentas para acesso à Internet de baixo custo terá efeitos importantes na educação odontológica no mundo todo. ${ }^{8}$

Tendo em sua fundamentação teórica, a aprendizagem como uma atividade de elaboração conceitual em um ambiente caracterizado pela interação social, o questionário COLLES foi escolhido, pois foi projetado para monitorar as práticas de aprendizagem online e verificar na medida em que estas práticas se configuram como processos dinâmicos favorecidos pela interação. Através do COLLES os tutores podem monitorar as perspectivas dos alunos sobre o ambiente de aprendizagem online contrastadas com sua prática nas interações e desta forma podem investigar como suprir as falhas de um determinado programa de educação à distância. ${ }^{17}$

Os resultados do presente estudo revelaram excelente relevância em que 42 alunos $(73 \%)$ relatam quase sempre a importância do aprendizado para prática da profissão e 39 alunos (68\%) demonstraram a melhorar no seu desempenho. Já em relação à reflexão crítica, $41 \%$ refletem criticamente com frequência sobre os conteúdos do curso e $43 \%$ refletem sobre como está sendo o seu aprendizado.
A interatividade entre os alunos apresenta-se deficiente, pois uma pequena parcela $(21 \%)$ se dispõe a explicar frequentemente suas ideias aos outros participantes e $48 \%$ dos alunos às vezes pedem explicações sobre as ideias. Em outro estudo, a implementação do ambiente de aprendizagem Moodle aumentou a aceitação entre os alunos, sem se encontrar diferenças entre sexos. No entanto a postagem do material educativo no ambiente virtual não influenciou a colaboração entre os mesmos. ${ }^{13}$

De acordo com a Muirhead \& Juwah (2003), pesquisas apontam que o acesso a atividades que levem alunos a refletir sobre conceitos tanto teóricos como baseados em sua própria experiência estabelecendo representações, questionamentos, diálogos, análises são fundamentais para a qualidade da interação, atuando como suporte para o processo de aprendizagem em nível epistemológico, já que levam alunos a refletir sobre conceitos tanto teóricos como baseados em sua própria experiência. Um aspecto relacionado ao enriquecimento do processo de aprendizagem é o uso de recursos tecnológicos e de multimídia. ${ }^{12}$

Com relação ao apoio dos tutores, os resultados 
Avaliação da percepção dos alunos da disciplina de endodontia sobre o uso do Ambiente Virtual de Aprendizagem (Moodle).

Uso do questionário de auto-avaliação COLLES • Cunha-Araújo IMZ, Salazar-Silva JR, D’Assunção FLC, Melo ABP

demonstram que frequentemente o tutor estimula a refletir $(47,3 \%)$, encoraja a participar $(40,3 \%)$ e melhora a reflexão autocrítica do participante $(45,6 \%)$. Já no apoio entre os colegas, houve variações entre as repostas apresentando baixa frequência de elogio $(26,3 \%)$ e estima as contribuições $(19,2 \%)$. Os alunos também apresentaram frequente compreensão das mensagens dos participantes $(50,8 \%)$ e dos tutores $(57,8 \%)$.

$\mathrm{O}$ EaD puro sofre muitas críticas quanto à motivação do aluno inserido em seu contexto, principalmente dirigidas à ausência do professor. Na realidade, quando se desenvolvem cursos em EaD, observa-se que o aprendizado pode ocorrer à distância, desde que o professor esteja completamente presente, ainda que virtualmente, ou seja, o contato do professor com seus alunos deve ser constante dentro das ferramentas que assim o permitam. ${ }^{16}$

Óbvio está que a complementação do ensino presencial com suporte a distância soma vantagens de ambos os aspectos educacionais, porém ainda necessita de um material desenvolvido com o intuito de motivar o aluno ao estudo individual para que ocorra o desempenho almejado. Os estudos de interatividade e "design" instrucional, além da usabilidade das ferramentas propostas, devem ser levados em consideração no momento da adequação do material a ser disponibilizado pelo corpo docente. ${ }^{16}$

\section{CONCLUSÕES}

Considerando a qualidade da interação um dos principais parâmetros para avaliação de ambientes de aprendizagem online, conclui-se que apesar dos bons índices de compreensão das mensagens, reflexão sobre os conteúdos e apoio dos tutores, existe necessidade de estimular o apoio entre os alunos e interação de suas ideias para que haja um melhor aproveitamento dentro do meio de ensino à distância.

\section{ABSTRACT}

Assessing how endodontics students perceive the Virtual Learning Environment (Moodle) with the COLLES self-assessment questionnaire

The discipline of Endodontics I taught at the Federal University of Paraíba (UFPB) introduced the Learning Management System by Virtual Learning Environment (Moodle) as of the class of 2008 $\left(1^{\text {st }}\right.$ sem. $)$. When students finish the course, they are required to answer to a self-assessment questionnaire available in the Moodle environment. The aim of this study was to analyze the results of the COLLES
(Constructivist On-Line Learning Environment Survey) questionnaire-an "effective experience" type questionnaire-applied to the 57 students from the classes of 2008 ( $2^{\text {nd }}$ sem.) and 2009 ( $1^{\text {st }}$ sem.) of the discipline of Endodontics I. COLLES evaluates the quality of the learning process in the virtual environment, according to the following perceptions: Professional Relevance, Reflective Thinking, Interactivity, Cognitive Demand, Affective Support and Interpretation of Meaning. Based on the answers, tables were constructed with results in numbers that were later analyzed quantitatively. The results indicated a high relevance rate, in that $73.6 \%$ of the students reported that what they learned was important to professional practice, and $68.4 \%$ reported having improved their own performance. In relation to Reflective Thinking, $41 \%$ of the subjects frequently reflect critically on the subject content, and $43.8 \%$ reflect on how their learning process is developing. The interactivity among the students was considered poor, because only a small proportion (21\%) was willing to explain their ideas to other participants. As for Cognitive Demand, students were often encouraged to reflect and engage in participation. In relation to Affective Support, there was a low frequency of acknowledgment and valuation of the contributions offered. The students were frequently found to understand the messages of participants $(50.8 \%)$ and tutors $(57.8 \%)$. Based on the results of the present research, it was concluded that, for the Virtual Learning Environment to be best taken advantage of, it is necessary to encourage support among students and interaction of their ideas.

\section{DESCRIPTORS}

Education, Distance. Teaching Materials. Teaching. Dental Education. Endodontics. -

\section{REFERÊNCIAS}

1. Abreu MC, Masetto MT. O professor Universitário em Aula: prática e princípios teóricos. 8 ed. São Paulo: MG Editores Associados, 1990.

2. Al-Jewair TS, Qutub, AF, Malkhassian G. et al. (2010). A Systematic Review of Computer-Assisted Learning in Endodontics Education. J Dent Educ: (74) 6: 601-611

3. Brasil. Ministério da Educação e Cultura. Conselho Nacional de Educação. Câmara de Educação Superior. Parecer CNE/ CES 1300/2001. Diretrizes Curriculares Nacionais dos Cursos de Graduação em Odontologia [acesso 25 nov 2010] Disponível em: http://www.abeno.org.br

4. Brasil. Ministério da Educação. Gabinete do Ministro. Porta- 
Avaliação da percepção dos alunos da disciplina de endodontia sobre o uso do Ambiente Virtual de Aprendizagem (Moodle).

Uso do questionário de auto-avaliação COLLES • Cunha-Araújo IMZ, Salazar-Silva JR, D’Assunção FLC, Melo ABP

ria 4.059/2004. Diário Oficial da União n 238, 13.12.2004, Seção 1, página 34

5. Cunha ALL, Quirino, ACC, Melo ABP, Salazar-Silva JR. A Avaliação do processo ensino-aprendizagem nas atividades práticas da disciplina Endodontia I da UFPB. In: XI Encontro de Iniciação à Docência, 2008, João Pessoa. Anais/Catálogo de Resumos - X ENEX / XI ENID, 2008.

6. Cunha IMZ, Salazar-Silva JR. Avaliação do ensino presencial e a distância na Disciplina de Endodontia. Int J Dent, 8 (supl 1): 12. 2009.

7. Fernandes APS. Modelo de um ambiente educacional via web para Odontologia - um estudo de caso em Periodontia [Tese de Doutorado]. Florianópolis: Universidade Federal de Santa Catarina; 2001.

8. Hillenburg KL, Cederberg RA, Gray SA, et al. (2006) E-learning and the future of dental education: opinions of administrators and information technology specialists. Eur J Dent Educ; 10 (3): 169-177

9. INEP. Instituto Nacional de Estudos e Pesquisas Educacionais Anísio Teixeira. Censo da educação superior 2010 - resumo técnico. 2012. 85 p.

10. Masetto MT. Aulas Vivas. 3 ed. São Paulo: MG Editores Associados, 1992.

11. Mattheos N, Schoonheim-Klein M, Walmsley AD, et al. (2010). Chapple LC. Innovative educacional methods and technolo- gies applicable to continuing professional development in periodontology. Eur J Dent Educ 14 (Suppl. 1) 43 -52.

12. Muirhead B, Juwah C. (2004). Interactivity in computer-mediated college and university education: a recent review of the literature. Educational Technology \& Society, 7 (1), 12-20.

13. Pahinis K, Stokes CW, Walsh TF, et al. (2008). A Blended Learning Course Taugth to Different Groups of Learners in a Dental School: Follow-Uo Evaluation. J Dent Educ: (72) 9: $1048-1057$.

14. Piletti C. Didática geral. São Paulo: Ática. 1987.

15. Primo LPCA. (2004). "Metodologia para acompanhamento de cursos de EAD e avaliação de competências - A2COMP”. Dissertação de Mestrado defendida em 1/Dezembro/2004. p. 145. Universidade de Fortaleza.

16. Skelton-Macedo MC, Basilio CC, Alves NCC, et. al. Endodontia na graduação com ensino presencial e suporte a distância: estratégia motivacional ao estudo individual. Revista ABENO. v.7, n. 1, p. 68-75, 2007.

17. Taylor PC, Dougiamas, M, Maor D [Internet]. Perth, Western Australia; Curtin University of Technology; c 2000 [citado abril 2010]. Disponível em: http://surveylearning.moodle. com/

Recebido em 08/10/2012

Aceito em 10/12/2012 